\title{
ARTICLE
}

\section{Alterations in functional brain networks in depressed patients with a suicide attempt history}

\author{
JeYoung Jung ${ }^{1}$, Sunyoung Choi ${ }^{2}$, Kyu-Man Han ${ }^{3}$, Aram Kim ${ }^{4}$, Wooyoung Kang ${ }^{4}$, Jong-Woo Paik ${ }^{5}$, Hae-Woo Lee ${ }^{6}$ and Byung-Joo Ham ${ }^{3}$
}

\begin{abstract}
Suicide is a major challenge in public health and is strongly associated with major depressive disorder (MDD). Despite recent neuroimaging developments, the neural correlates of suicide behavior in patients with MDD remain unclear. Independent component analysis (ICA) for neuroimaging data allows the identification of functional brain networks without prior regions of interest and may help to identify neurobiological markers of specific disorders. Using ICA, we investigated the differences in restingstate brain networks in patients with MDD who had or did not have a history of suicide attempts and in healthy controls (HCs). Suicidal depressed (SD) patients, non-suicidal depressed (NSD) patients, and HCs significantly differed from each other in the pattern of connectivity of multiple functional networks, network synchronization, and functional network connectivity (FNC). The patient groups had a decreased network synchronization in the insular, cerebellum, basal ganglia, thalamus, operculum, frontoparietal cortices, and sensory cortices relative to the HCs. The decreased FNC between these networks (insular-default mode network and insular-cerebellum) was found in the SD group compared to the NSD and HC groups. These differences were not related to illness duration and medication status differences between SD and NSD. Furthermore, the degree of FNC in these networks was associated with the suicide ideation and stress level. Our results demonstrated that widespread but discrete network changes in brain networks and their interconnectivity was associated with suicide attempts in patients with MDD. Our results suggest that the neural basis underlying the psychopathology of attempted suicide in patients with MDD involves multiple brain networks and their interaction.
\end{abstract}

Neuropsychopharmacology (2020) 45:964-974; https://doi.org/10.1038/s41386-019-0560-z

\section{INTRODUCTION}

Suicide is a major challenge for today's healthcare systems. One person dies from suicide every $40 \mathrm{~s}$, and one million people die from suicide every year, accounting for a global mortality rate of 16 per 100,000 [1]. Several risk factors have been found to contribute to suicidal behavior and ideation such as prior and current history of medical conditions and psychosocial states $[2,3]$. Among them, mental disorder is a major risk factor for suicide, with major depressive disorder (MDD) as one of the leading causes of suicide, comprising half of all people who died by suicide [4]. Indeed, one study reported that $58 \%$ of patients with MDD had suicidal ideation and 15\% of patients attempted suicide [5]. Despite of the significant link between MDD and suicide, the underlying neural basis of suicide attempts in patients with MDD remains unclear.

To better understand the neural mechanisms underlying suicide attempts in patients with MDD, researchers have investigated structural and functional brain alterations in patients with MDD who have attempted suicide compared with those of healthy persons and/or patients with MDD who have not attempted suicide $[6,7]$. Imaging studies have reported structural abnormalities in cortical and subcortical regions, including the basal ganglia $[8,9]$, amygdala, orbitofrontal cortex (OFC) [10], cerebellum [11], dorsomedial prefrontal cortex (PFC), insular, and midbrain [12], as well as the fronto-striato-limbic network [13] and temporal-parietal-limbic system [14]. Functional imaging studies have shown that suicide attempters have functional alterations in the ventral, medial, and lateral PFC $[15,16]$, basal ganglia, limbic system [16], and thalamus [17]. Cognitively, suicide attempters showed impaired executive function, attention, and memory, which is linked to prefrontal lobe dysfunction [18-21]. These findings suggest that suicidality in MDD is associated with widespread structural and functional regional alterations in the brain that are associated with impaired functions.

Resting-state functional magnetic resonance imaging (rsfMRI) studies have been used to explore the intrinsic activity or restingstate brain networks (RSNs), such as the default mode network (DMN), which has been reported to be altered in various psychopathological conditions [22]. Few studies have investigated the resting-state activity or the RSNs in suicide attempters with depression and have reported inconsistent results about the brain areas associated with the resting-state functional alterations [23]. rsfMRI studies have demonstrated synchronous alterations in intrinsic activity of the frontal, temporal, and parietal areas in

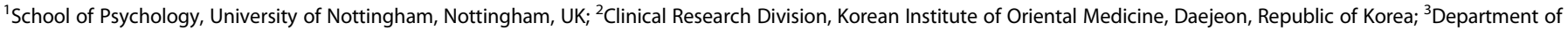

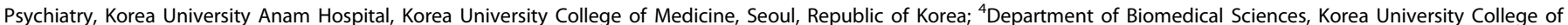

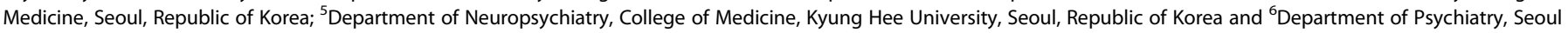
Medical Center, Seoul, Republic of Korea

Correspondence: Byung-Joo Ham (hambj@korea.ac.kr)

These authors contributed equally: JeYoung Jung, Sunyoung Choi

Received: 23 May 2019 Revised: 19 September 2019 Accepted: 8 October 2019

Published online: 31 October 2019 
suicide attempters [24, 25]. Zhang et al. [26] found that both depressed suicidal and non-suicidal adolescents exhibited an increased functional connectivity within the $\mathrm{DMN}$, relative to healthy controls (HCs). A recent study reported that suicide attempters had increased functional connectivity of the amygdala with the insular, OFC, and middle temporal gyrus (MTG) compared with that of non-suicide attempters. The increased connectivity between the amygdala and parahippocampal region in suicide attempters was correlated with suicidal ideation [27]. Another study investigated suicidal ideation in functional connectivity of patients with MDD. They demonstrated decreased connectivity in the fronto-thalamic circuit in patients with suicidal ideation relative to patients without it [28]. Although rsfMRI studies have revealed functional alterations in various brain areas and in the DMN, it is not yet known how suicidality in MDD is associated with various brain networks beyond the DMN and their interactions.

Here we compared the resting-state functional brain networks between the patients with depression who had attempted suicide, patients with depression who had not attempted suicide, and HCs. To define RSNs, we used independent component analysis (ICA), which is a data-driven multivariate approach to delineate spatially and temporally independent components (networks) [29]. Based on previous findings, we hypothesized that, compared with nonsuicidal depressed (NSD) patients and HCs, suicidal depressed (SD) patients would show altered patterns of neural activity in multiple RSNs as well as a different functional network connectivity (FNC) between them. We also hypothesized that the NSD group would show abnormal changes in RSNs and FNC relative to the HC group. Moreover, we predicted that these abnormal changes in RSNs would be significantly associated with the scores of the clinical scales or questionnaire that assess suicidal behavior and stress.

\section{MATERIALS AND METHODS}

Participants

We recruited 61 patients with MDD from the outpatient psychiatry clinic at the Korea University Anam Hospital, Guro Hospital, Ansan Hospital, Kyunghee University Medical Center, and Seoul Medical Center in Seoul, the Republic of Korea between March 2015 and November 2016. We included patients aged 19-60 years who had been diagnosed with MDD by board-certified psychiatrists (Ham $\mathrm{B}-\mathrm{JH}, \mathrm{J}-\mathrm{WP}$, and $\mathrm{H}-\mathrm{WL}$ ) using a structured clinical interview from the Diagnostic and Statistical Manual of Mental Disorders, Fourth Edition Axis I disorders. Our exclusion criteria for patients with MDD or bipolar disorder (BD) were as follows: (i) any other major psychiatric comorbidity, including personality or substance use disorders; (ii) current psychotic features (e.g., delusions or hallucinations); (iii) history of serious and unstable medical illness; (iv) primary neurological illness, including head trauma with residual effects; and (v) any contraindication to MRI scan, such as metal implants or claustrophobia. A total of 61 patients with MDD were divided into two groups: 31 with SD who had previously attempted suicide and 30 NSD who had no previous history of any suicide attempts. Suicide attempt history was confirmed by a clinical interview and based on the documentation of suicide attempts in their medical records. The Risk-Rescue Rating (RRR) scale was administered to patients with SD to assess the lethality of current or most recent suicide attempt [26]. Illness duration was also assessed in patients with MDD using the life-chart methodology, psychotropic medication history, and their current status. Illness duration was defined as the elapsed time since the patient had experienced their first mood episode, regardless of inter-episodic periods. Thirty-two HCs, also aged between 19 and 60 years, were recruited from the community via an advertisement. Board-certified psychiatrists performed full psychiatric assessments to confirm that none of the HCs currently or previously had any psychopathology. The exclusion criteria applied to the patient groups were also applied to the $\mathrm{HC}$ group.
All patients' (SD and NSD) and HCs' severity of depressive symptoms was measured by the 17-item Hamilton Depression Rating Scale (HDRS) [30] on the day of MRI. We confirmed that no patients (both SD and NSD groups) were refractory to the pharmacological treatment at the time of MRI scan and according to the treatment failure criteria at least two trials of antidepressants of different classes were given at adequate doses [31]. To evaluate the severity of suicidal ideation and impulsiveness, participants were asked to complete a self-report version of the Scale for Suicide Ideation (SSI) [32] and the Barratt Impulsiveness Scale (BIS) [33] on the day of the MRI scan. The self-report Toronto Alexithymia Scale (TAS-20) scale was used to measure alexithymia [34]. The Perceived Stress Scale (PSS) was implemented to measure the degree to which situations in one's life were considered stressful [35]. The 17-item HDRS is a clinicianadministered 17-item questionnaire used to measure of the severity of depressive symptoms based on probing mood, feelings of guilt, suicide ideation, insomnia, agitation or retardation, anxiety, weight loss, and somatic symptoms [30]. The SSI is a self-report 19-item clinical research instrument designed to quantify and assess suicidal intention. Generally, the SSI score is correlated with clinical ratings of suicidal risk and selfadministered measure of self-harm [32]. The BIS is a widely used self-report measure of impulsiveness and designed to assess the personality/behavioral construct of impulsiveness [33]. TAS-20 is a self-report 20-item instrument that is one of the most commonly used measures of alexithymia, which identifies individuals having trouble identifying and describing emotions and tended to minimize emotional experience and focus attention externally [34]. PSS is one of the most widely used 10-item psychological instruments to measure the extend of stressful situations in one's life [35].

The study's protocol was approved by the Institutional Review Board of the Korea University Anam Hospital. In accordance with the Declaration of Helsinki, all participants gave their written informed consent prior to participation in the study.

\section{Statistical analysis}

Differences in demographics between the SD patients, NSD patients, and HCs were analyzed using a one-way analysis of variance (Scheffe's test as the post hoc analysis, $p<0.05$ ) for continuous variables (age, years of education, and HDRS scores), and $x^{2}$ test for sex. Analysis of covariance (ANCOVA) was used to calculate between-group differences in questionnaire scores (BIS, PSS, and TAS-20 scores), including age as a covariate. A $t$ test was performed to compute differences in illness duration between the SD and NSD groups. All statistical analyses were performed using IBM SPSS Statistics for Windows, version 24.0 (IBM Corporation, Armonk, NY, USA).

rsfMRI acqusition

A 3-T Siemens scanner was used to acquire imaging data in the Korea University Magnetic Resonance Imaging Centre. Participants were instructed to keep their eyes open during the scan and to stare passively at a fixation cross (6 min, 180 volume). See Supplementary Material for the detailed imaging parameters.

Multivariate analysis-ICA

Preprocessing was performed via CONN (https://www.nitrc.org/ projects/conn). The rsfMRI data were realigned to generate six rigid-body parameters characterizing the estimated subject motion. Based on realignment, data that showed $>2 \mathrm{~mm}$ and/or $2^{\circ}$ head motion were excluded from this study. Then data were coregistered with their anatomical images, spatially normalized to the Montreal Neurological Institute space, and spatially smoothed using a Gaussian kernel ( $8 \mathrm{~mm}$, full-width half-maximal). In order to remove motion, physiological, and other artifactual effects, denoising was conducted using CompCor [36]. Data were 
detrended, despiked, and filtered with a band pass filter $(0.01<<$ 0.08 ) to decrease the effects of low-frequency drift and influence of potential outlier scans. ICA was performed using the group ICA of fMRI Toolbox (GIFT) [37]. The pre-processed data were entered into the GIFT version 3.0b. The toolbox concatenates the individual data followed by the computation of subject-specific components and time course. Using principal component analysis, individual data were reduced. The informax algorithm [38] was applied for the group ICA and estimated 22 components. To improve the independent component's stability, ICASSO was applied and run 20 times [39].

Of the 22 components, 6 were related to residual artifacts, including the signal distributed around the edge of the brain and within the cerebrospinal fluid spaces. Sixteen components were correlated with the brain and defined as RSNs (Fig. 1a). For the selected RSNs, we considered the two following outcome variables: (1) the component power spectra and (2) the FNC. Details of the processing flows are described in Supplementary Material.

Our primary aim was to detect neural differences between the three groups (HC, NSD, and SD). For the selected RSNs, we directly compared each group with another using $t$ tests in SPM12 (Wellcome Department of Imaging Neuroscience, London; www. fil.ion.ucl.ac.uk/spm). The resulting whole-brain maps were thresholded at $p<0.005$ at the voxel level with False Discovery Rate (FDR)-corrected cluster threshold of $p<0.05$, ks $>100$. Two outcomes from the ICA analysis were compared between groups using ANCOVA with covariates including age and years of education followed by post hoc $t$ test $(p<0.05)$. For the comparison between the patients' groups, a separate post hoc analysis was performed with illness duration and medication use treatment (antidepressant-naive $=0$; taking antidepressant $=$ 1) as covariates. The outcome measures were correlated with questionnaire scores (SSI, BIS, PSS, and TAS-20) using Pearson's correlations with age and years of education as covariates $\left(p_{\text {FDR-corrected }}<0.05\right)$. Details of the FDR correction are described in Supplementary Material.

\section{RESULTS}

Demographics, clinical scale scores, and questionnaire scores No significant differences were found in age and sex between the three groups (Table $1 ; p>0.1$ ). Years of education were significantly different between the three groups $\left(F_{2,90}=19.998\right.$, $p<0.001)$. Post hoc $t$ test revealed that the $\mathrm{HC}$ group had significantly more years of education than both the SD $(p<0.001)$ and NSD groups $(p<0.001)$, but there was no significant difference between the two MDD groups $(p>0.1)$. Illness duration was significantly different between the NSD and SD groups $(p<$ 0.05).

The HDRS and SSI scores were significantly different between the three groups (HDRS score: $F_{2,90}=198.1, p<0.001$; SSI score: $\left.F_{2,90}=60.179, p<0.001\right)$. Post hoc $t$ test revealed that the $\mathrm{HC}$ group had a significantly lower HDRS score than the SD $(p<0.001)$ and NSD groups $(p<0.001)$, but there was no significant difference between the two MDD groups. Post hoc $t$ test also revealed that the SD group had significantly higher SSI scores than both the HC $(p<0.001)$ and NSD groups $(p<0.001)$, and the NSD group had a higher SSI score than the HC group $(p<0.001)$.

The BIS, PSS, and TAS-20 scores were significantly different between the three groups (BIS score: $F_{2,90}=6.146, p<0.05$; PSS score: $F_{2,90}=110.892, p<0.001$; TAS-20 score: $F_{2,90}=38.514, p<$ $0.001)$. Post hoc $t$ test revealed that the SD group had significantly higher BIS scores than the HC group $(p<0.05)$ as well as higher PSS and TAS-20 scores than the NSD group (PSS: $p<0.001$; TAS-20: $p<0.001)$ and HC group (PSS: $p<0.001$; TAS-20: $p<0.001$ ). The NSD group had higher PSS scores than HCs (PSS: $p<0.001$; TAS-20: $p<0.05)$.

\section{RSNs and power spectra}

ICA estimated the 16 components showing patterns of temporally coherent signals confined to the brain, which were considered as the RSNs. We labeled the RSNs according to their spatial location (e.g., visual network) or their previously described labels (e.g., DMN). Figure 1a and Table S1 show the spatial map of the selected RSNs.

First, we directly compared each of the RSNs between the groups. Figure 1 and Table S2 present between-group differences in RSNs. Compared with the HC, the MDD (NSD+SD) group showed a reduced connectivity in several RSNs, including CO4 (insular network: IN), C05 (DMN), C06 (cerebellum network: CN), C11 (thalamus-cerebellum network: TCN), C14 (medial PFC network: mPFCN), C16 (dorsal attention network: DAN), and C22 (sensory-motor network: SMN) (Fig. 1b). The IN (CO4) included the bilateral insular and anterior cingulate cortex (ACC). In C04 (IN), the MDD group showed reduced connectivity in the right superior frontal gyrus (SFG) and cerebellum than that in the HC group. The DMN (C05) consisted of the MPFC, precuneus, posterior cingulate cortex (PCC), and angular gyrus. In C05 (DMN), MDD showed a significantly reduced connectivity in the superior medial gyrus and anterior/middle/posterior cingulate cortex (ACC/MCC/PCC). The $\mathrm{CN}$ (C06) included the bilateral cerebellum and exhibited reduced connectivity in the OFC in MDD patients relative to HCs. The basal ganglia network (BGN: C09) consisted of the thalamus and basal ganglia. Patients showed decreased connectivity in the thalamus as compared with that in the HCs. TCN (C11) included the thalamus, cerebellum, midbrain, and insular. In C11 (TCN), the SD group showed decreased connectivity in the lingual gyrus and right middle frontal gyrus (MFG). The MPFCN (C14) consisted of the ACC and OFC. MDD patients had decreased connectivity in the right temporal pole and cerebellum as well as increased connectivity in the cuneus, right inferior frontal gyrus (IFG) and precentral/postcentral gyrus as compared with that in the $\mathrm{HC}$ group. The DAN included the bilateral SFG, MFG, superior parietal lobe (SPL), inferior parietal lobe, precuneus, and inferior temporal gyrus. C16 (DAN) showed a significantly decreased connectivity in the posterior medial frontal cortex in the MDD group. The SMN (C22) included the bilateral primary motor cortex, primary sensory cortex, and SPL, and the MDD group showed reduced connectivity in the left MFG and insular as well as enhanced connectivity in the MCC, thalamus, and MTG as compared to that in the HC group.

We compared the RSNs between the NSD and SD groups (Fig. 1C). C04 (IN) showed an increased connectivity in the right putamen in the SD group as compared to that in the NSD group. C09 (BGN) showed a reduced connectivity in the PCC in the SD group as compared to that in the NSD group. In addition, the SD group exhibited an enhanced connectivity in the thalamus of C22 (SMN) as compared to that in the NSD group.

Figure 2 shows the result of power spectra analysis of each network. The primary visual network (CO3: VN1) included the bilateral primary visual cortex and showed a significant main effect of group $\left(F_{2,88}=5.33, p<0.01\right)$ in the power spectra. Post hoc tests revealed that the NSD group had a lower power than the $\mathrm{HC}$ and SD group. No significant difference was observed between the $\mathrm{HC}$ and SD groups. The power of the IN (C04) showed a significant effect of group $\left(F_{2,88}=3.12, p<0.05\right)$. Post hoc tests revealed that the network power was decreased in the both NSD and SD groups as compared to the HC group. CN (C06) also showed a group effect $\left(F_{2,88}=3.20, p<0.05\right)$ in the power spectra. Post hoc tests revealed that the network power was decreased in the NSD and SD groups as compared to the HC group. C09 (BGN) showed a significant main effect of group $\left(F_{2,88}=4.37, p<0.05\right)$ in the power spectra. The NSD and SD groups had a significant reduction in the network power as compared to the HC group. No difference was observed between the NSD and SD groups. The power spectra of $\mathrm{C} 11$ (TCN) showed a significant main effect of group $\left(F_{2,88}=5.52, p<0.01\right)$. Post hoc tests revealed that the 
A C03 Primary Visual Network (VN1)

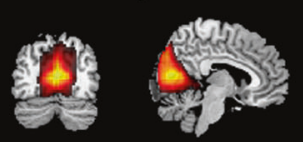

CO4 Insular Network (IN)
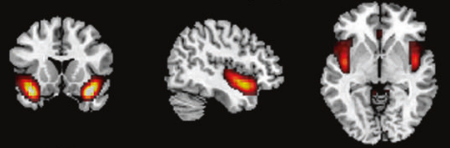

C05 Default Mode Network (DMN)
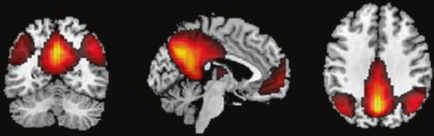

C06 Cerebellum Network (CN)

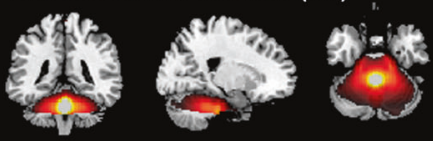

C09 Basal Ganglia Network (BGN)

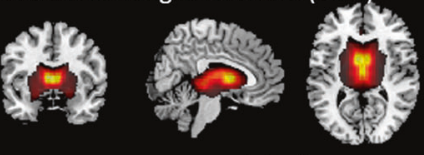

C10 Semantic Network (SN)
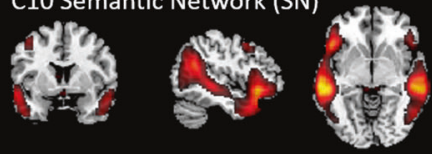
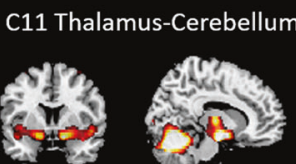

C12 Ventro-Temporal Network (vTN)
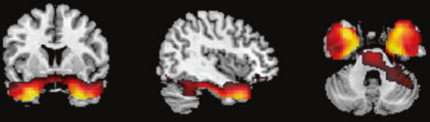

C13 Visual Network (VN2)
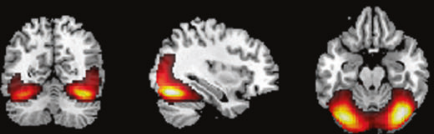

C14 Medial Prefrontal Cortex Network (mPFCN)

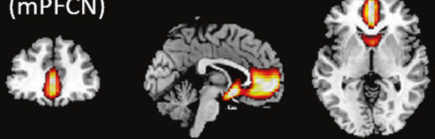

C16 Dorsal Attention Network (DAN)
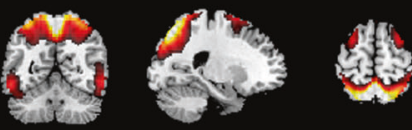

C17 Right Fronto-Parietal Network (rFPN)
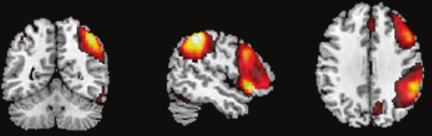

B

HC vs MDD (NSD +SD)

C04 IN

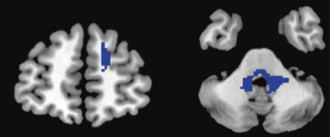

C05 DMN

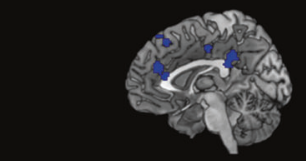

$\mathrm{C06} \mathrm{CN}$

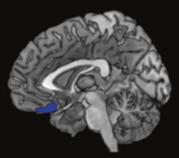

CO9 BGN

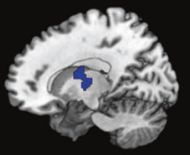

C11 TCN

- $\mathrm{HC}>\mathrm{MDD}$

$M D D>\mathrm{HC}$

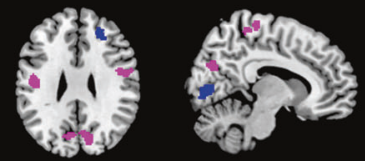

C14 mPFCN
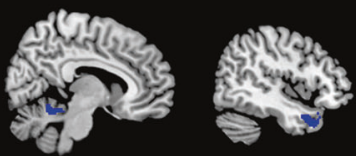

C16 DAN

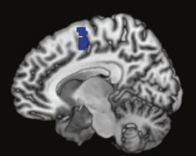

C22 SMN

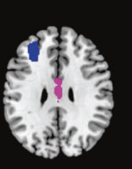

C18 Left Fronto-Parietal Network (IFPN)

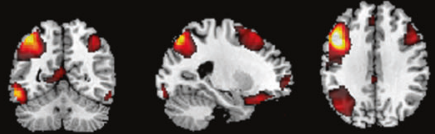

C19 Operculum Network (ON)

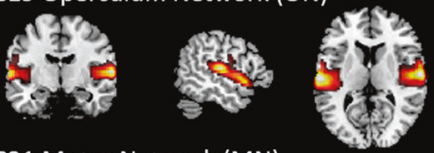

C21 Motor Network (MN)

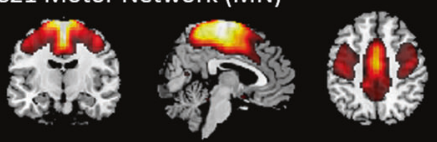

C22 Sensory-Motor Network (SMN)

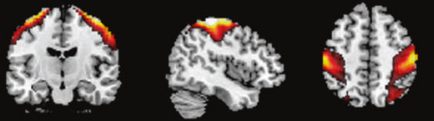

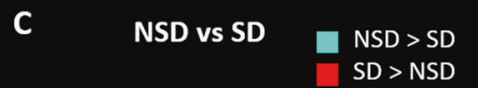

CO4 IN

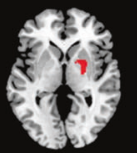

CO9 BGN

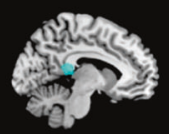

C22 SMN

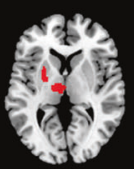

Fig. 1 a The spatial map of 16 components (RSN) derived from the ICA analysis. The statistical threshold was set at $T>20$. See Table for coordinates. b Comparisons in RSNs between HC and MDD (NSD+SD). Compared with the HC, patients with MDD showed reduced connectivity in several RSNs, including C04 (IN), C05 (DMN), C06 (CN), C09 (BGN), C11 (TCN), C14 (mPFCN), C16 (DAN), and C22 (SMN) as well as increased connectivity in C11 (TCN) and C22 (SMN). Blue indicates regions showing decreased functional connectivity in the MDD group as

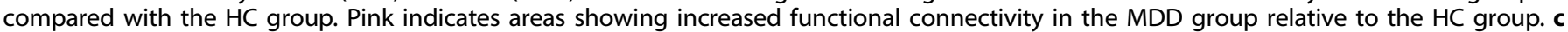
Comparisons in RSNs between NSD and SD. Compared with the NSD group, SD group showed decreased connectivity in C09 (BGN) as well as increased connectivity in C04 (IN) and C22 (SMN). Cyan indicates regions which showed that the functional connectivity was lower in the SD group than those in the HC group. Red indicates areas that showed increased functional connectivity in the SD group relative to the NSD group 
J Jung et al.

Table 1. Demographic information, clinical characteristics, and questionnaire scores of the three groups and between-group comparisons

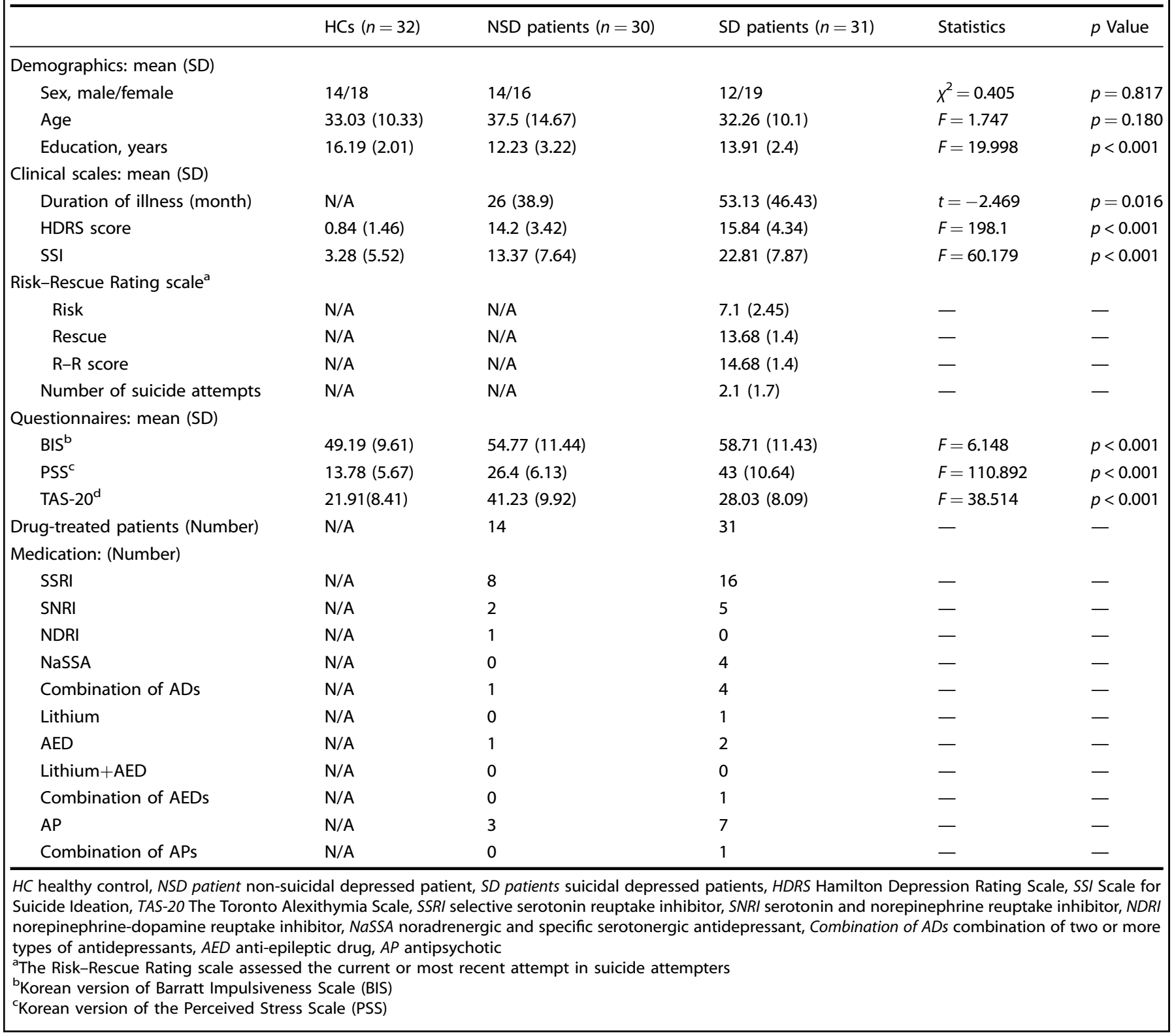

network power was significantly decreased in the NSD and SD groups as compared to the HC group. The mPFC network (mPFCN: C14) consisted of MPFC and ACC. The secondary visual network (C13) consisting of the lateroventral visual cortices showed the significant group effect $\left(F_{2,88}=4.06, p<0.05\right)$. The network power of $C 14$ showed the main effect of the group $\left(F_{2,88}=4.66, p<0.05\right)$. Post hoc tests revealed that the network power was significantly decreased in the SD group as compared to the HC group. The power spectra of C16 (DAN) exhibited a significant group difference $\left(F_{2,88}=4.61, p<0.05\right)$. Post hoc tests revealed that the NSD group has decreased network power as compared to that of the HC group. No significant difference was observed between the $\mathrm{SD}$ and $\mathrm{HC}$ groups. The network power of operculum network (C19) including the bilateral operculum showed the significant group effect $\left(F_{2,88}=3.67, p<0.05\right)$ revealing the decreased power of it in both the NSD and SD groups relative to the HC group. We found the group difference in the power spectra of the motor network (C21) consisted of the bilateral primary motor cortex and supplementary motor area $\left(F_{2,88}=3.60, p<0.05\right)$. Post hoc tests revealed that the network power was significantly decreased in the NSD and SD groups as compared to the HC group. The power spectra of C22 (SMN) revealed the significant group effect $\left(F_{2,88}=\right.$ $3.12, p<0.05)$, showing the reduced power in the NSD group compared to the $\mathrm{HC}$ group.

Functional network connectivity

Figure $3 a$ shows the FNC results for each RSN. We found a significant effect of the group on the FNC between the several RSNs (Table S3). Post hoc tests were performed for these networks and the results are displayed in Fig. 3b. Compared to the $\mathrm{HC}$ group, the NSD group exhibited a decreased FNC in $\mathrm{CO3}-\mathrm{C13}$, C09-C11, and C14-C19, as well as an increased FNC in C03-C06, C03-C11, C04-C06, C11-C13, C11-C21, and C16-C17. Compared to the HC group, the SD group exhibited a decreased FNC in C04-C05 and C14-C19, as well as an increased FNC in C04-C06. Compared to the NSD group, the SD group exhibited a decreased FNC in C03-C06, C03-C11, C04-C05, C05-C11, C06-C16, C11-C13, $\mathrm{C} 11-\mathrm{C} 21$, and $\mathrm{C} 16-\mathrm{C} 17$, as well as an upregulated $\mathrm{FNC}$ in $\mathrm{C} 03-\mathrm{C} 13$. 

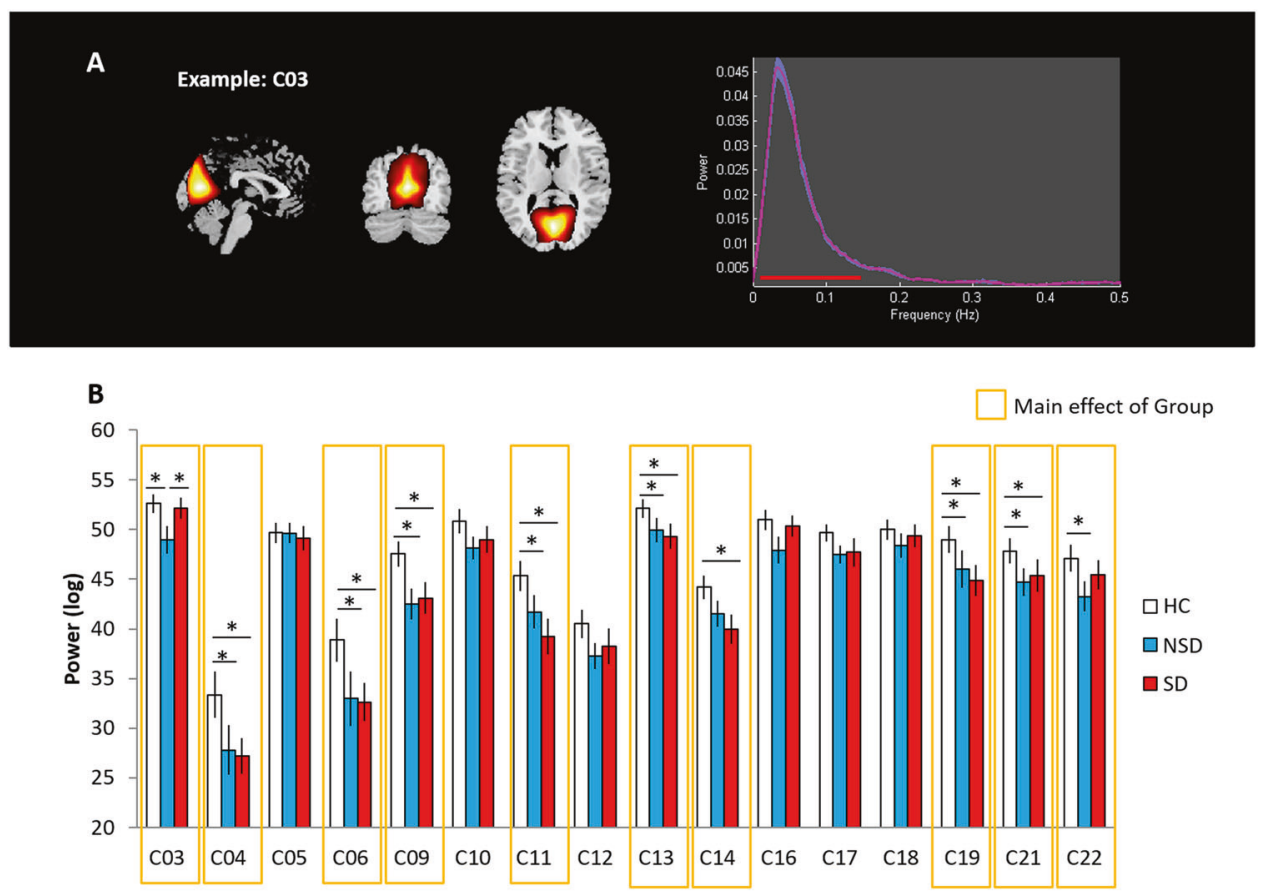

Fig. 2 The network power spectra. a An example of power spectra, C03 (VN1). The sum of low frequency fluctuation power (red line, $0.01-0.15 \mathrm{~Hz}$ ) was used to determine the strength of the signal of interest within the RSNs. $\mathbf{b}$ Group differences in power spectra. The yellow boxes indicate the RSN that showed a significant group effect $(p<0.05)$. The white bar represents the HC group, the blue bar represents the NSD group, and the red bar indicates the SD group. Error bars indicate the standard error $\left({ }^{*} p<0.05\right)$

A correlation analysis was used to investigate the relationship between the FNC and the questionnaire scores. The FNC between C04 and C05 gradually decreased from the HC to NSD groups and from the NSD to SD groups; the HC group showed no connectivity, whereas the NSD and SD groups showed decoupling in C04-C05 (Fig. 3c). The FNC in C04-C05 was negatively correlated with the SSI scores (All: $r=-0.25, p_{\text {FDR-corrected }}<0.05$; Patients: $r=-0.28$, $p_{\text {FDR-corrected }}<0.05$ ) and PSS scores (All: $r=-0.30, p_{\text {FDR-corrected }}<$ 0.005; Patients: $\left.r=-0.34, p_{\text {FDR-corrected }}<0.01\right)$. By contrast, the inter-network connectivity between $\mathrm{C} 04$ and C06 showed the opposite pattern; the $\mathrm{HC}$ group exhibited the strongest decoupling, and the degree of connectivity reduced gradually from the NSD group to the SD group (Fig. 3d). The FNC in C04-C06 was positively correlated with the SSI scores (All: $r=0.34, p_{\text {FDR-corrected }}$ $<0.05$; Patients: $r=0.22, p_{\mathrm{FDR} \text {-corrected }}<0.05$ ) and PSS scores (All: $r=0.31, \quad p_{\text {FDR-corrected }}<0.005$; Patients: $r=0.30, p_{\text {FDR-corrected }}<$ 0.01). Patients with a weaker decoupling in this FNC had higher SSI and PSS scores. The inter-network connectivity between C09 and $\mathrm{C} 11$ was reduced in the NSD group compared to the HC group and was negatively correlated with TAS-20 scores (All: $r=$ $-0.34, p_{\text {FDR-corrected }}<0.001$; Patients: $r=-0.28, p_{\text {FDR-corrected }}<$ 0.05) (Fig. 3e). No other correlations reached statistical significance.

Furthermore, the FNC in C06-C22 and C14-C16 in the SD group showed a significant correlation with PSS score (Fig. 4). In the SD group, the FNC between C06 and C22 was positively correlated with the PSS score $\left(r=0.45, p_{\text {FDR-corrected }}<0.05\right)$, but this was not the case in the other groups (Fig. 4a). The interconnectivity between C14 and C16 was negatively correlated with the PSS score in the SD group $\left(r=-0.47, p_{\text {FDR-corrected }}<0.05\right)$ (Fig. 4b). No significant correlations were found in the other two groups.

\section{DISCUSSION}

Recently, Northoff [40] suggested that various depressive symptoms can be translated as intrinsic activity in spatiotemporal disturbances, which has been supported by recent findings that link abnormal spatial organization of the resting-state activity to ruminations and increased self-focus in depression. A metaanalysis of rsfMRI in patients with MDD compared to the HCs showed the hyperactivity in the medial prefrontal regions, thalamus, midbrain, and cerebellum as well as hypoactivity in the insular, ACC, PCC, precuenus/cuneus, temporal pole, caudate, cerebellum, and dorsolateral PFC (DLPFC) [41]. The authors argue that the contrasting results in these regions illustrated the disbalance between the two networks with abnormal changes in the DMN (anterior and posterior midline regions) and central executive network (CEN; DLPFC). These findings are corroborated by dysfunctional mood and emotional regulation in $\operatorname{MDD}[42,43]$, supporting Northoff's suggestion [40]. Similarly, our comparisons between the MDD and $\mathrm{HC}$ groups in RSNs demonstrated the altered functional connectivity in these regions in IN, DMN, CN, BGN, TCN, mPFCN, DAN, and SMN as well as deceased network synchronization in these networks. Moreover, our results demonstrated that the direction of these alterations (increasing or decreasing functional connectivity) were dependent on the network. Specifically, the anterior and posterior midline regions showed decreased functional connectivity with the DMN, CN, and DAN, whereas increased connectivity with the TCN and SMN. These results are compatible with a recent view on the functional heterogeneity of DMN and CEN that can be reorganized depending on specific demands by fractionizing itself or recruiting additional regions $[44,45]$. Our findings may illustrate the complex network dynamics derived from deficits in emotional processing in MDD beyond the imbalance between the DMN and CEN.

Based on these findings in MDD, Serafini et al. [23] proposed that vulnerability to suicidal behavior can be attributed to increased self-focus and hopelessness derived from emotional dysregulation in combination with abnormalities in the frontolimbic or fronto-parietal-cerebellar pathways and DMN. Concordantly, decreased network synchronization as well as different patterns of connectivity in the IN, CN, BGN, TCN, and mPFCN were found in the SD group. Moreover, the SD group showed more alterations in the IN, BGN, and SMN as compared to the NSD 


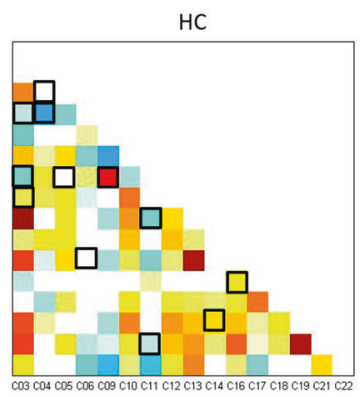

B
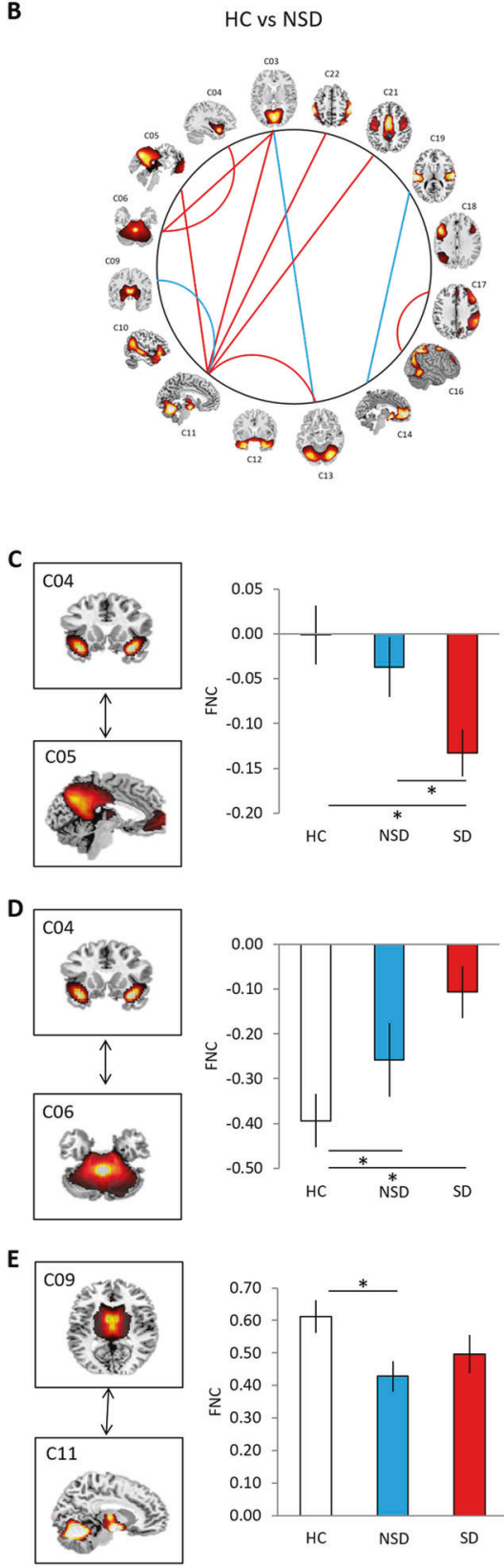
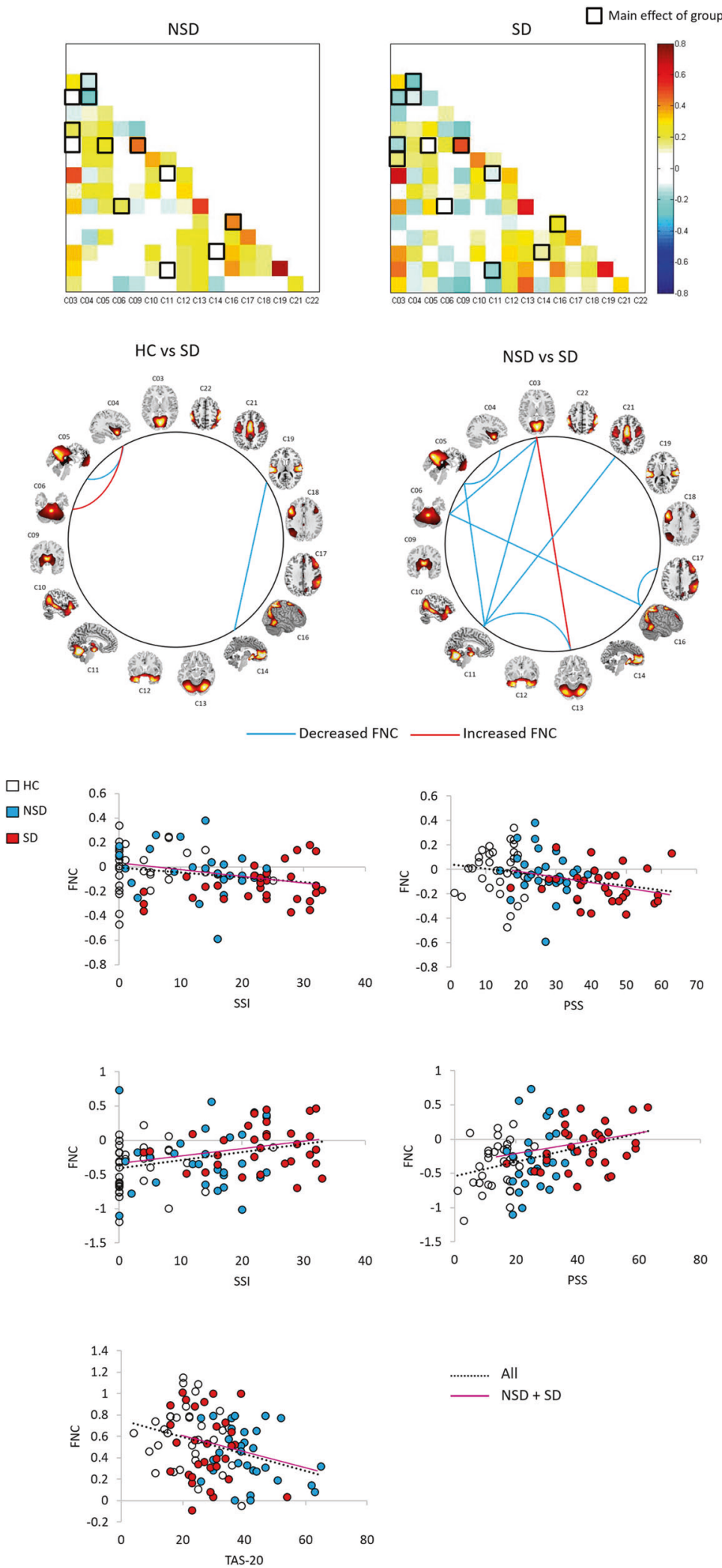

Fig. 3 Functional network connectivity (FNC) between RSNs. a FNC matrix. Pairwise correlations between RSN time courses were Fisher $z-$ transformed and averaged across subjects within the HC, NSD, and SD groups. The black boxes indicate FNC that showed a significant group effect. $\mathbf{b}$ Group differences in the selected FNC. The blue line indicates a decreased FNC and the red line indicates an increased FNC ( $p<0.05)$. c The relationship between the FNC and behavioral measures. The FNC between C04 (IN) and C05 (DMN). d The FNC between C04 (IN) and C06 (CN). e The FNC between C09 (BGN) and C11 (TCN). White bars and circles represent the HC, blue ones represent the NSD, and red ones represent the SD. Error bars indicate the standard error. Dotted lines indicate the correlation of all participants and pink lines patients. ${ }^{*} p<$ 0.05 

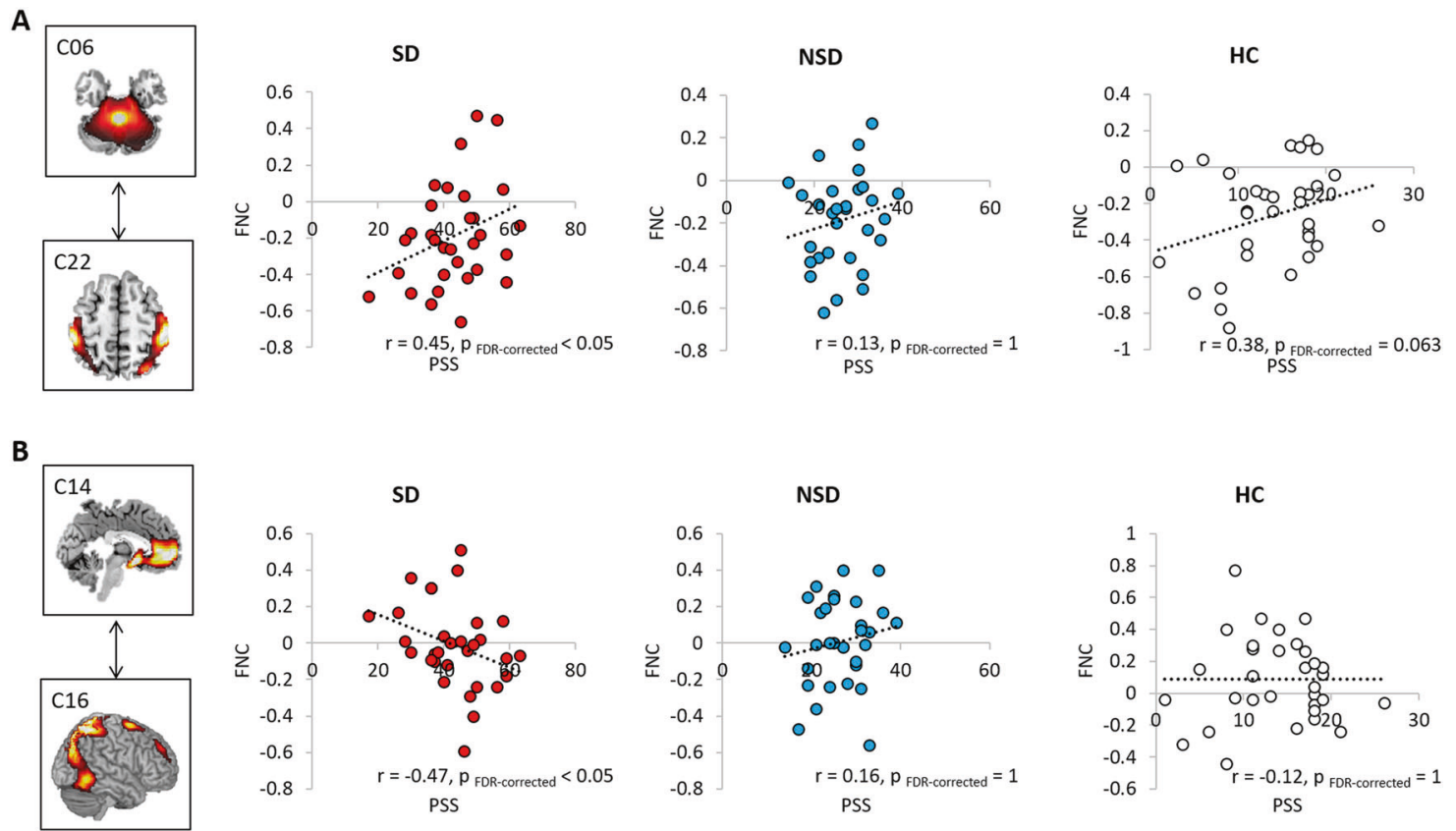

Fig. 4 The relationship between the stress level and FNC according to groups (SD, NSD, and HC). a The FNC between C06 (CN) and C22 (SMN). The SD group showed a significant correlation between them, demonstrating that SD patients with higher stress level exhibited stronger connectivity between C06 and C22. b The FNC between C14 (mPFCN) and C16 (DAN). The SD group revealed a significant negative correlation between them. SD patients with higher stress level showed less connectivity between the C14 and C16. Red circles represent the SD group, blue represents the NSD group, and white represents the HC group

group: increased connectivity with the putamen in the IN and the thalamus in the SMN and decreased connectivity with the PCC in the BGN. Enhanced connectivity in limbic regions may contribute to emotional dysregulation by biasing the sensory stimuli processing [46], whereas decoupling in the PCC as part of the DMN may be attributed to self-oriented processing in the SD group [23]. These results support Serefini et al.'s view on abnormalities in RSNs related to suicidality.

The novel finding of the current study is that the SD group had a decreased inter-network connectivity between the IN and DMN compared with the NSD and HC groups. The insular is widely connected with subcortical and cortical regions and plays a crucial role in various functions, including emotion cognition, selfawareness, interpersonal experience, and sensory perception [47-49]. Previous studies in patients with depression have reported structural and functional abnormalities in the insular, which could be a marker of vulnerability to depression [50-53]. Furthermore, the insular is a key node of the salience network (SN), which is involved in the detection and integration of emotional and sensory stimuli and in modulating the switch between the DMN and CEN [54]. Previous studies have reported an aberrant structure and function of the SN, DMN, and CEN in patients with MDD [55-57]. In particular, evidence has suggested that patients with depression exhibit abnormalities within the SN and a dysfunctional interaction of the SN with other intrinsic networks such as the DMN and CEN [53, 55, 58, 59]. Here we demonstrated a reduced network synchronization of the IN in both SD and NSD groups relative to the HC group, but only the SD group showed significantly decreased inter-network connectivity changes between the IN and DMN compared to the other groups. Furthermore, individuals with more decoupling between the networks showed higher suicide ideation and stress levels. Our findings suggest that dysfunctional relationships between these networks may contribute to the vulnerability to suicidal behavior and could represent a potential marker for suicidal behavior in MDD.
SD patients showed differential alterations in the $I N-C N$ connectivity. The cerebellum plays an important role in motor control, cognition, and emotional processing and is involved in a variety of psychiatric disorders, including depression, BD, and schizophrenia [60]. Suicidal behavior in those with depression has been associated with a decreased cerebellar volume [12] and low regional cerebral blood flow in the cerebellum [16]. Similarly, we found that the SD group had a lower network synchronization in the $\mathrm{CN}$. Also, the more abnormal alterations in these inter-network connectivity, the higher the suicide ideation and stress level. Overall, the FNC alterations of these networks (IN-DMN and IN-CN) could reflect an impaired internal processing related to the suicidality in depressive patients.

These findings also have implications for theoretical perspectives of suicidal behavior. Theories of suicide span from biological (e.g., dysregulation of the serotonergic system in the ventromedial PFC), psychodynamic (e.g., distributed attachment and intense affective states), cognitive-behavioral (e.g., hopelessness and emotion dysregulation) to developmental/ systems etiologies (e.g., disrupted social forces and family). In an effort to integrate diverse suicide theories, Van Orden and colleagues [61] proposed the interpersonal theory of suicide having three constructs: two related to suicidal desirethwarted belongingness and perceived burdensomeness and the acquired capability for suicide. The current results suggest that patients with SD had the decreased network function in various systems associated with cognitive control, decisionmaking, emotion regulation, and sensory processing and dysfunctional network interactions in the IN-DMN and IN-CN contributing to suicidal ideation and perceived stress. These neural alterations may underpin various clinical features of patients with SD such as hopelessness and self-blame [23], which can form thwarted belongingness and perceived burdensomeness responding to life events (e.g., unemployment, psychical illness, loss through death/divorce, and childhood abuse) [61]. Our results provide the neural mechanism 
underpinning suicidality in MDD proposing that network-level alterations may play a role in developing suicide desires suggested by the current theory of suicide behavior.

The current understanding of suicidal behavior is based on a stress-vulnerability model-individuals are at higher risk of committing suicide in stressful situations, and the vulnerability to suicidal behavior has been modeled in emotion and cognitive impairments mediated by PFC [62]. Specifically, suicidality has been reportedly associated with reduced volume in the ventral and dorsal PFC $[12,63]$, lower glucose uptake in the ventral PFC [15], and prefrontal lobe dysfunction when engaging in cognitive tasks [64]. Based on these findings, Jollant et al. [63, 65] have suggested a neuroanatomical model accounting for the vulnerability to suicidal behavior implicated by the ventral PFC in valuation deficits-decision-making impairments in patients with SD [66], whereas the dorsal parts of the PFC are associated with cognitive control and emotion regulation [67]. Consistently, the SD group was consistently found to have lower network power as compared with HCs in the mPFCN consisting of ventral (OFC) and dorsal (ACC) prefrontal regions. Thus reduced mPFC network power may explain emotion regulation and cognitive control deficits in patients with SD, making them more vulnerable to suicidal behavior. Together, our findings suggest that PFC dysfunction may represent a neuroanatomical marker of suicidality in patients with MDD.

And interestingly, suicide attempters with stronger network connectivity between the mPFCN and DAN showed lower stress scores. The DAN is involved in mediating the top-down guided voluntary allocation of attention to stimuli [68]. It is possible that suicide attempters with a relatively intact DAN can mediate the impaired PFC functions by biasing stimuli processing, resulting in lower stress scores. It should be noted that there was no difference in the DAN power between the SD and HC groups. Pan et al. [69] showed that adolescents with a history of depression and suicide attempts showed no abnormalities in performance accuracy and attentional control network activity on tasks of cognitive control. Our finding suggests that suicide attempters might be able to regulate their stress through the crosstalk between the preserved DAN and impaired mPFCN.

The current study has several limitations. First, its sample size is relatively small. Second, we could not fully control the differences between SD and NSD patients in the antidepressant treatment and illness duration. The antidepressant treatment in patients with MDD showed heterogeneous concomitant medication types, dosage, and durations and therefore could represent potential confounding factors. In addition, the illness duration in SD patients was significantly longer than that in NSD patients; most SD patients were revisiting outpatients, whereas half of NSD patients were new patients who were antidepressant drug-naive. Although we accounted for the medication use and illness duration in our analysis, we could not exclude the possibility that they might affect our findings. Third, we did not evaluate the change of depressive symptoms severity according to antidepressant treatment longitudinally, thus we could not obtain the information about whether the patients were non-responsive to treatment (e.g., antidepressant response on 8- or 4-week treatment). Therefore, we could not exclude the possibility that refractory nature of MDD, which has been reported to be associated with functional brain network and suicidality, may affect our results [70, 71]. Fourth, the $H C$ group had longer education years than those in both the SD and NSD groups. In order to test the effect of longer education, we re-analyzed the data by splitting the HC group as lower and higher education groups and found no significant effect of years of education in the RSN and FNC. Also, there is a possibility that the disease itself may interfere with the educational process, leading to relatively shorter education years in patients. Furthermore, rsfMRI can be confounded by the effects of head motion during data collection.
Although our analysis controlled for motion-related confounds, it may not yet be sufficient to provide full control of them. In order to better to control it, newer approach such as real-time correction methods or framewise displacement should be considered in the future. Finally, suicidal behavior has been repeatedly associated with cognitive dysfunction [67, 72]; however, the association among history of suicidal attempt, cognitive performance, and RSN/FNC was not investigated in this study. Future studies with larger sample sizes and a well-defined patient population will better elucidate the underlying neural mechanisms of suicide behaviors and will help explore complex relationships between suicidality, cognitive function, and brain networks.

\section{CONCLUSION}

To our best knowledge, this study is the first study to examine multiple brain networks and their interactions using the ICA approach in depressed patients who have attempted suicide. We demonstrated that widespread but discrete network changes in functional networks and their interconnectivity were associated with a predisposition to suicide. Our results collaborate previous findings and are compatible with the abnormality in cognitive and affective profiles of suicide attempters. Our findings support Serafini et al.'s view [23] of the relationship between fMRI abnormalities and vulnerability to suicide behavior and extend our understanding of the neural substrates of suicide in MDD.

\section{FUNDING AND DISCLOSURE}

This work was supported by the University of Nottingham's research fellowship programme to JJ, the Research Fund (14-BR-SS-03) of Civil-Military Technology Cooperation Program, the Civil-Military Dual-Use Technology Deveploment Program (14-BR-SS-03) throught the Agency for Defense Development, and the Bio \& Medical Technology Development Program of the National Research Foundation (NRF) funded by the Ministry of Science, ICT \& Future Planning (NRF-2016M3A9A7916996; NRF-2017R1A2B4002090) to B$\mathrm{JH}$. The authors declare no competing interests.

\section{ADDITIONAL INFORMATION}

Supplementary Information accompanies this paper at (https://doi.org/10.1038/ s41386-019-0560-z).

Publisher's note Springer Nature remains neutral with regard to jurisdictional claims in published maps and institutional affiliations.

\section{REFERENCES}

1. WHO. First WHO report on suicide prevention. 2014. http://www.who.int/ mediacentre/news/releases/2014/suicide-prevention-report/en/.

2. Pompili M, Venturini $P$, Lamis DA, Giordano G, Serafini G, Belvederi Murri $M$, et al. Suicide in stroke survivors: epidemiology and prevention. Drugs Aging 2015;32:21-9.

3. Joiner TE Jr., Brown JS, Wingate LR. The psychology and neurobiology of suicidal behavior. Annu Rev Psychol. 2005;56:287-314.

4. Seguin M. Suicide Risk Management: A Manual for Health Professionals, 2nd edition. Canadian Psychology-Psychologie Canadienne. 2012;53:255-7.

5. Sokero TP, Melartin TK, Rytsala HJ, Leskela US, Lestela-Mielonen PS, Isometsa ET. Suicidal ideation and attempts among psychiatric patients with major depressive disorder. J Clin Psychiatry. 2003;64:1094-100.

6. Desmyter S, van Heeringen C, Audenaert K. Structural and functional neuroimaging studies of the suicidal brain. Prog Neuropsychopharmacol Biol Psychiatry 2011;35:796-808.

7. van Heeringen $K$, Bijttebier $S$, Desmyter $S$, Vervaet $M$, Baeken $C$. Is there a neuroanatomical basis of the vulnerability to suicidal behavior? A coordinate-based metaanalysis of structural and functional MRI studies. Front Hum Neurosci 2014;8:824.

8. Ahearn EP, Jamison KR, Steffens DC, Cassidy F, Provenzale JM, Lehman A, et al. MRI correlates of suicide attempt history in unipolar depression. Biol Psychiatry $2001 ; 50: 266-70$. 
9. Dombrovski AY, Siegle GJ, Szanto K, Clark L, Reynolds CF, Aizenstein H. The temptation of suicide: striatal gray matter, discounting of delayed rewards, and suicide attempts in late-life depression. Psychol Med 2012;42:1203-15.

10. Monkul ES, Hatch JP, Nicoletti MA, Spence S, Brambilla P, Lacerda ALT, et al. Fronto-limbic brain structures in suicidal and non-suicidal female patients with major depressive disorder. Mol Psychiatry 2007;12:360-6.

11. Lee YJ, Kim S, Gwak AR, Kim SJ, Kang SG, Na KS, et al. Decreased regional gray matter volume in suicide attempters compared to suicide non-attempters with major depressive disorders. Compr Psychiatry 2016;67:59-65.

12. Hwang JP, Lee TW, Tsai SJ, Chen TJ, Yang CH, Lirng JF, et al. Cortical and subcortical abnormalities in late-onset depression with history of suicide attempts investigated with MRI and voxel-based morphometry. J Geriatr Psychiatry Neurol. 2010;23:171-84.

13. Wagner G, Koch K, Schachtzabel C, Schultz CC, Sauer H, Schlosser RG. Structural brain alterations in patients with major depressive disorder and high risk for suicide: evidence for a distinct neurobiological entity? Neuroimage 2011:54:1607-14.

14. Peng HJ, Wu K, Li J, Qi HC, Guo SW, Chi MY, et al. Increased suicide attempts in young depressed patients with abnormal temporal-parietal-limbic gray matter volume. J Affect Disord. 2014;165:69-73.

15. Oquendo MA, Placidi GPA, Malone KM, Campbell C, Keilp J, Brodsky B, et al. Positron emission tomography of regional brain metabolic responses to a serotonergic challenge and lethality of suicide attempts in major depression. Arch Gen Psychiatry. 2003;60:14-22.

16. Amen DG, Prunella JR, Fallon JH, Amen B, Hanks C. A comparative analysis of completed suicide using high resolution brain SPECT imaging. J Neuropsychiatry Clin Neurosci. 2009;21:430-9.

17. Audenaert K, Peremans K, Goethals I, van Heeringen C. Functional imaging, serotonin and the suicidal brain. Acta Neurol Belgica. 2006;106:125-31.

18. Keilp JG, Sackeim HA, Brodsky BS, Oquendo MA, Malone KM, Mann JJ. Neuropsychological dysfunction in depressed suicide attempters. Am J Psychiatry. 2001;158:735-41.

19. Jollant F, Lawrence NS, Giampietro V, Brammer MJ, Fullana MA, Drapier D, et al. Orbitofrontal cortex response to angry faces in men with histories of suicide attempts. Am J Psychiatry. 2008;165:740-8.

20. Reisch T, Seifritz E, Esposito F, Wiest R, Valach L, Michel K. An fMRI study on mental pain and suicidal behavior. J Affect Disord. 2010;126:321-5.

21. Jollant $F$, Lawrence NS, Olie E, O'Daly O, Malafosse A, Courtet $P$, et al. Decreased activation of lateral orbitofrontal cortex during risky choices under uncertainty is associated with disadvantageous decision-making and suicidal behavior. Neuroimage 2010;51:1275-81.

22. Buckner RL, Andrews-Hanna JR, Schacter DL. The brain's default network: anatomy, function, and relevance to disease. Ann NY Acad Sci. 2008;1124:1-38.

23. Serafini G, Pardini M, Pompili M, Girardi $P$, Amore $M$. Understanding suicidal behavior: the contribution of recent resting-state fMRI techniques. Front Psychiatry. 2016;7:69.

24. Cao J, Chen XR, Chen JM, Ai M, Gan Y, Wang W, et al. Resting-state functional MRI of abnormal baseline brain activity in young depressed patients with and without suicidal behavior. J Affect Disord. 2016;205:252-63.

25. Fan $\Pi$, Wu X, Yao L, Dong J. Abnormal baseline brain activity in suicidal and non-suicidal patients with major depressive disorder. Neurosci Lett 2013;534:35-40.

26. Zhang S, Chen JM, Kuang L, Cao J, Zhang H, Ai M, et al. Association between abnormal default mode network activity and suicidality in depressed adolescents. BMC Psychiatry 2016;16:337.

27. Kang SG, Na KS, Choi JW, Kim JH, Son YD, Lee YJ. Resting-state functional connectivity of the amygdala in suicide attempters with major depressive disorder. Prog Neuropsychopharmacol Biol Psychiatry 2017;77:222-7.

28. Kim K, Kim SW, Myung W, Han CE, Fava M, Mischoulon D, et al. Reduced orbitofrontal-thalamic functional connectivity related to suicidal ideation in patients with major depressive disorder. Sci Rep 2017;7:15772.

29. Calhoun VD, Adali T, Pearlson GD, Pekar JJ. A method for making group inferences from functional MRI data using independent component analysis. Hum Brain Mapp. 2001;14:140-51.

30. Hamilton M. A rating scale for depression. J Neurol Neurosurg Psychiatry. 1960;23:56-62.

31. Sackeim HA. The definition and meaning of treatment-resistant depression. J Clin psychiatry. 2001;62(Suppl 16):10-7.

32. Beck AT, Steer RA, Ranieri WF. Scale for Suicide Ideation: psychometric properties of a self-report version. J Clin Psychol. 1988;44:499-505.

33. Patton JH, Stanford MS, Barratt ES. Factor structure of the Barratt impulsiveness scale. J Clin Psychol. 1995;51:768-74.

34. Bagby RM, Parker JD, Taylor GJ. The twenty-item Toronto Alexithymia Scale-I. Item selection and cross-validation of the factor structure. J Psychosom Res. 1994;38:23-32.
35. Cohen S, Kamarck T, Mermelstein R. A global measure of perceived stress. Health Soc Behav. 1983;24:385-96.

36. Behzadi Y, Restom K, Liau J, Liu TT. A component based noise correction method (CompCor) for BOLD and perfusion based fMRI. Neuroimage 2007;37:90-101.

37. Egolf EA, Calhoun VD, Kiehl KA. Group ICA of fMRI Toolbox (GIFT). Biol Psychiat 2004;55:8S-8S.

38. Bell AJ, Sejnowski TJ. An information-maximization approach to blind separation and blind deconvolution. Neural Comput 1995;7:1129-59.

39. Himberg J, Hyvarinen A, Esposito F. Validating the independent components of neuroimaging time series via clustering and visualization. Neuroimage 2004;22:1214-22.

40. Northoff G. Spatiotemporal psychopathology I: No rest for the brain's resting state activity in depression? Spatiotemporal psychopathology of depressive symptoms. J Affect Disord. 2016;190:854-66.

41. Alcaro A, Panksepp J, Witczak J, Hayes DJ, Northoff G. Is subcortical-cortical midline activity in depression mediated by glutamate and GABA? A cross-species translational approach. Neurosci Biobehav Rev. 2010;34:592-605.

42. Mayberg HS. Modulating dysfunctional limbic-cortical circuits in depression: towards development of brain-based algorithms for diagnosis and optimised treatment. Br Med Bull. 2003;65:193.

43. Phillips ML, Drevets WC, Rauch SL, Lane R. Neurobiology of emotion perception II: Implications for major psychiatric disorders. Biol Psychiatry 2003;54:515-28.

44. Camilleri JA, Muller VI, Fox P, Laird AR, Hoffstaedter F, Kalenscher T, et al. Definition and characterization of an extended multiple-demand network. Neuroimage 2018;165:138-47.

45. Andrews-Hanna JR, Reidler JS, Sepulcre J, Poulin R, Buckner RL. Functionalanatomic fractionation of the brain's default network. Neuron 2010;65:550-62.

46. Lu Y, Liang H, Han D, Mo Y, Li Z, Cheng Y, et al. The volumetric and shape changes of the putamen and thalamus in first episode, untreated major depressive disorder. Neuroimage Clin 2016;11:658-66.

47. Craig AD. How do you feel? Interoception: the sense of the physiological con dition of the body. Nat Rev Neurosci. 2002;3:655-66.

48. Singer T, Critchley HD, Preuschoff K. A common role of insula in feelings, empathy and uncertainty. Trends Cogn Sci. 2009;13:334-40.

49. Augustine JR. Circuitry and functional aspects of the insular lobe in primates including humans. Brain Res Brain Res Rev. 1996;22:229-44.

50. Takahashi T, Yucel M, Lorenzetti V, Tanino R, Whittle S, Suzuki M, et al. Volumetric MRI study of the insular cortex in individuals with current and past major depression. J Affect Disord. 2010;121:231-8.

51. Jung J, Kang J, Won E, Nam K, Lee MS, Tae WS, et al. Impact of lingual gyrus volume on antidepressant response and neurocognitive functions in major depressive disorder: a voxel-based morphometry study. J Affect Disord 2014;169:179-87.

52. Wise T, Radua J, Via E, Cardoner N, Abe O, Adams TM, et al. Common and distinct patterns of grey-matter volume alteration in major depression and bipolar disorder: evidence from voxel-based meta-analysis. Mol Psychiatry 2017;22:1455-63.

53. Peng X, Lin P, Wu X, Gong R, Yang R, Wang J. Insular subdivisions functional connectivity dysfunction within major depressive disorder. J Affect Disord 2018:227:280-8.

54. Menon V, Uddin LQ. Saliency, switching, attention and control: a network model of insula function. Brain Struct Funct. 2010;214:655-67.

55. Menon V. Large-scale brain networks and psychopathology: a unifying triple network model. Trends Cogn Sci. 2011;15:483-506.

56. Hamilton JP, Furman DJ, Chang C, Thomason ME, Dennis E, Gotlib IH. Defaultmode and task-positive network activity in major depressive disorder: implications for adaptive and maladaptive rumination. Biol Psychiatry 2011;70:327-33.

57. Sheline YI, Barch DM, Price JL, Rundle MM, Vaishnavi SN, Snyder AZ, et al. The default mode network and self-referential processes in depression. Proc Nat Acad Sci USA. 2009;106:1942-7.

58. Kaiser RH, Andrews-Hanna JR, Wager TD, Pizzagalli DA. Large-scale network dysfunction in major depressive disorder: a meta-analysis of resting-state functional connectivity. JAMA Psychiatry 2015;72:603-11.

59. van Tol MJ, Veer IM, van der Wee NJ, Aleman A, van Buchem MA, Rombouts SA et al. Whole-brain functional connectivity during emotional word classification in medication-free major depressive disorder: abnormal salience circuitry and relations to positive emotionality. Neuroimage Clin 2013;2:790-6.

60. Phillips JR, Hewedi DH, Eissa AM, Moustafa AA. The cerebellum and psychiatric disorders. Front Public Health. 2015;3:66.

61. Van Orden KA, Witte TK, Cukrowicz KC, Braithwaite SR, Selby EA, Joiner TE Jr. The interpersonal theory of suicide. Psychol Rev 2010;117:575-600.

62. Mann JJ. Neurobiology of suicidal behaviour. Nat Rev Neurosci. 2003;4:819-28.

63. Ding Y, Lawrence N, Olie E, Cyprien F, le Bars E, Bonafe A, et al. Prefrontal cortex markers of suicidal vulnerability in mood disorders: a model-based 
Alterations in functional brain networks in depressed patients with a...

$J$ Jung et al.

974

structural neuroimaging study with a translational perspective. Transl Psychiatry 2015;5:e516.

64. Minzenberg MJ, Lesh TA, Niendam TA, Yoon JH, Rhoades RN, Carter CS. Frontal cortex control dysfunction related to long-term suicide risk in recent-onset schizophrenia. Schizophr Res 2014;157:19-25.

65. Jollant F, Lawrence NL, Olie E, Guillaume $S$, Courtet $P$. The suicidal mind and brain: a review of neuropsychological and neuroimaging studies. World J Biol Psychiatry. 2011;12:319-39.

66. Richard-Devantoy S, Berlim MT, Jollant F. A meta-analysis of neuropsychological markers of vulnerability to suicidal behavior in mood disorders. Psychol Med 2014;44:1663-73.

67. Richard-Devantoy S, Jollant F, Kefi Z, Turecki G, Olie JP, Annweiler C, et al. Deficit of cognitive inhibition in depressed elderly: a neurocognitive marker of suicidal risk. J Affect Disord. 2012;140:193-9.
68. Vossel S, Geng JJ, Fink GR. Dorsal and ventral attention systems: distinct neural circuits but collaborative roles. Neuroscientist 2014;20:150-9.

69. Pan LA, Batezati-Alves SC, Almeida JR, Segreti A, Akkal D, Hassel S, et al. Dissociable patterns of neural activity during response inhibition in depressed adolescents with and without suicidal behavior. J Am Acad Child Adolesc Psychiatry. 2011;50:602.e3-11.e3.

70. Bergfeld IO, Mantione M, Figee M, Schuurman PR, Lok A, Denys D. Treatmentresistant depression and suicidality. J Affect Disord. 2018;235:362-7.

71. Dichter GS, Gibbs D, Smoski MJ. A systematic review of relations between restingstate functional-MRI and treatment response in major depressive disorder. J Affect Disord. 2015;172:8-17.

72. Richard-Devantoy S, Berlim MT, Jollant F. Suicidal behaviour and memory: a systematic review and meta-analysis. World J Biol Psychiatry. 2015;16:544-66. 